\title{
Industrial Application of Mechanical Reduction on Continuous Casting of Bearing Steel Bloom
}

\author{
Xinghua Chen, Wei Deng and Shuai Niu * \\ Special Steel Business Unit, Nanjing Iron \& Steel Co., Ltd., Nanjing 210035, China; \\ chenxinghua@njsteel.com.cn (X.C.); dengwei1@njsteel.com.cn (W.D.) \\ * Correspondence: niushuai@njsteel.com.cn; Tel.: +86-183-5186-1101
}

check for

updates

Citation: Chen, X.; Deng, W.; Niu, S. Industrial Application of Mechanical Reduction on Continuous Casting of Bearing Steel Bloom. Processes 2021, 9, 2280. https://doi.org/10.3390/ pr9122280

Academic Editor: Peng Lan

Received: 1 December 2021

Accepted: 17 December 2021

Published: 20 December 2021

Publisher's Note: MDPI stays neutral with regard to jurisdictional claims in published maps and institutional affiliations.

Copyright: (c) 2021 by the authors. Licensee MDPI, Basel, Switzerland. This article is an open access article distributed under the terms and conditions of the Creative Commons Attribution (CC BY) license (https:/ / creativecommons.org/licenses/by/ $4.0 /)$.
Abstract: Industrial experiments of mechanical soft reduction in continuous casting were conducted in the present study aiming to improve the internal quality of the bearing steel blooms. Two methods were developed to verify the solidification model for a reliable crater end in the caster, which is provided by SMS CONCAST. The verified solidification model was applied to determine the solidification status of the bloom and provides theoretical reduction region. Several trials were conducted to study the optimization of the reduction rate regarding the V-shaped and centerline segregation of the bloom. The results show an obvious improvement of internal quality in the bearing steel bloom by applying appropriate reduction during casting.

Keywords: continuous casting; bearing steel; mechanical reduction; V-shaped segregation

\section{Introduction}

The internal quality of the bearing steel bloom has great influence on its reliability and service life due to the inheritance of the defects from the bloom, such as shrinkage porosity, center segregation, and V-shaped segregation to the subsequent rolling product. Generally, homogenization of the chemical composition by means of electromagnetic stirring (EMS), pulsed magneto-oscillation (PMO), low superheat casting, low secondary cooling intensity, etc., is the most well-known approach to minimize the center segregation [1-6]. This approach aims to produce large ratio of equiaxed grains during solidification and normally is an effective way to reduce center segregation. However, coarse equiaxed grain can also cause the formation of bridging, and the enriched liquid will be partly sucked from interdendritic areas downwards to compensate the shrinkage at the final stage of solidification, and these compensated regions are the origins of the spot segregation [7-9]. Moreover, insufficient compensation of the solidification shrinkage between the settling of equiaxed crystals also causes the suction of enriched liquid when strand contraction occurs, and thus the V-shaped segregation is generated [10]. Compared to the methods mentioned above, applying mechanical soft reduction (MSR) in the final stage of solidification of the crater end, which has been successfully applied in continuous cast slab for improving the center segregation and the $\mathrm{V}$-shaped segregation, offers a possible alternative for producing low-segregation-level steel blooms, especially for the high-carbon and large-section steel blooms [11,12]. The MSR principle relies on its efficient compensation of the solidification shrinkage in the final state of solidification and preventing the suction flow to occur $[13,14]$. The determination accuracy of the position of the mushy region of the bloom and the reduction region and its amount is the critical precondition for the success of the application of the MSR process. Regarding the complicated industrial casting conditions, although several researchers had proposed empirical reduction amount/rate and region for different steel grades and bloom thickness, it is still difficult to form the common criteria for the industrial application of MSR [15-17].

Additionally, while the MSR is usually applied in the mushy region of the bloom, the mechanical heavy reduction (MHR) is applied in the totally solidified region immediately 
behind the crater end. The reduction amount of a single roller in the MHR unit can normally reach 10 20 $\mathrm{mm}$ or more. With this large reduction amount (similar to the rough rolling process), the compactness of the bloom core can be improved during continuous casting. Thus, the extent of the center porosity can be reduced. Regarding the advantage of the MHR for the compactness of the bloom core, all the MSR trials were conducted with a certain amount of MHR in the practiced trials. However, the optimization of the MHR was not discussed in this paper, as the MHR application position is far behind the mushy region and could not affect the compensation during solidification. The applied MHR values were provided only for reference.

The objective of the present work mainly focuses on the optimization of the MSR parameters to improve the internal quality of the bearing steel bloom by using the solidification model provided by SMS CONCAST. After the accuracy of the solidification model was verified, extensive industrial trials were designed and conducted based on the simulated solidification crater end at different casting conditions to study the effect of MSR.

\section{Crater End Determination and Experimental Setup}

\subsection{Model Validation}

The position of the crater end and mushy zone region were calculated by using the solidification model provided by SMS CONCAST. The MSR and MHR parameters need to be determined based on the simulated solid fraction of the bloom center. Thus, it is critical to verify the model before the optimization of the reduction parameters. Nail shooting is the most well-known method to estimate the position of solidification crater and then verify the solidification model; however, due to the regulation of bullet control, this method is prohibited in China. In order to verify the solidification model, two alternative methods were practiced in the present study, with medium-carbon and high-carbon steel, respectively.

One method involves measuring the position of white band [18] in bloom cross section. Figure 1 shows the verification result for medium-carbon steel. The hot etched cross section of a 45\# steel shows an obvious white band in the center region of the sample due to strong stirring by final electromagnetic stirring (F-EMS). The inner side of the white band corresponds to the solidification front which has a local solid fraction (fs) of 0.3 [19]. The measured and calculated distance from the inner side of the white band to bloom surface is $92.8 \mathrm{~mm}$ (average value) and $90.6 \mathrm{~mm}$, respectively. The calculation results agree well with the white band experiment results, indicating a certain accuracy of the simulation model for medium-carbon steels.

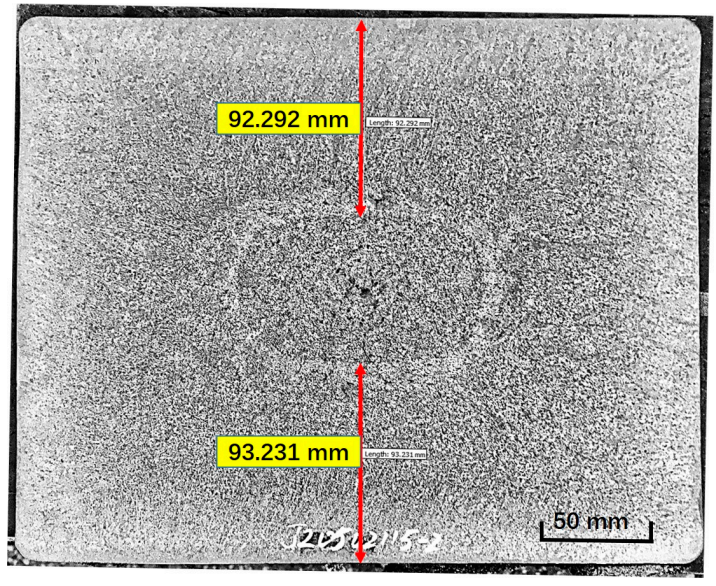

(a)

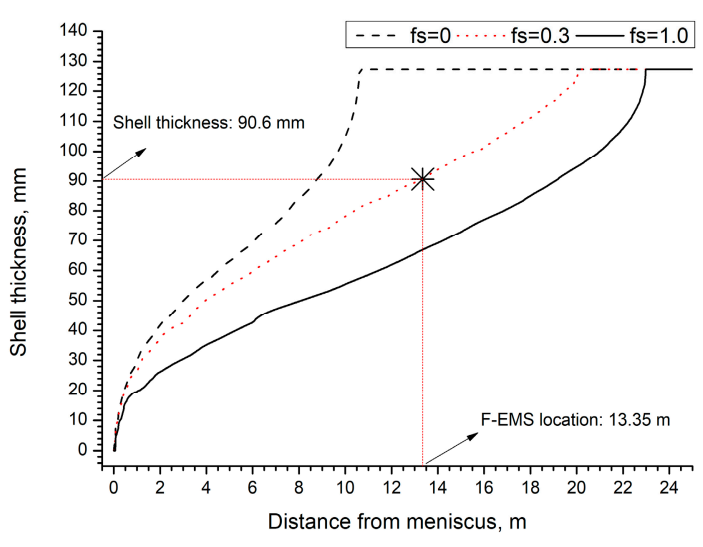

(b)

Figure 1. White band verification method result (steel 45): (a) Experimental and (b) simulated white band position. 
Differently to medium-carbon steels, the solute wash phenomenon generated by EMS is not obvious when dealing with high-carbon steels. An alternative model verification method for high-carbon steel (bearing steel) was developed based on the inner crack formation mechanism. The temperature range between zero-strength temperature (ZST, corresponds to solid fraction $\mathrm{fs}=0.8$ ) to zero-ductility temperature (ZDT, corresponds to solid fraction $\mathrm{fs}=1.0$ ) is considered as the brittle temperature range $[20,21]$. Any supercritical loading of the pinch roll within this range would lead to open internal cracks. Meanwhile, applying reduction in the region where the solid fraction of the bloom center has the range between 0.3 to 0.9 would result in a certain improvement of the V-shaped segregation $[7,15,22]$. Thus, comparing the inner crack occurrence position and the improvement extent of the V-shaped segregation to the calculated bloom solidification status at the positions of the pinch rolls would provide evidence of whether the model is accurate or not.

Based on the principle mentioned above, the verification trial for high-carbon steel GCr15 by applying "heavy reduction" during casting was conducted in NISCO, as shown in Figure 2a. Each reduction mark corresponds to only one withdrawal and straightening (WS) unit. The applied mechanical reduction force on WS1 WS4 and WS5 WS7 is $800 \mathrm{kN}$ and $1500 \mathrm{kN}$, respectively. The reduction amount has a variation range from 14 to $40 \mathrm{~mm}$. After the bloom was cooled down to room temperature, longitudinal section (the center section along bloom wideness direction) samples at the middle of the bloom along casting direction, also shown in Figure 2b, were cut out, grinded, and then immerged into a $60 \sim 70{ }^{\circ} \mathrm{C}$ etchant which is a mixture of industrial $\mathrm{HCl}$ and water with the ratio of 1:1 for 15 20 min to proceed the hot acid etching.

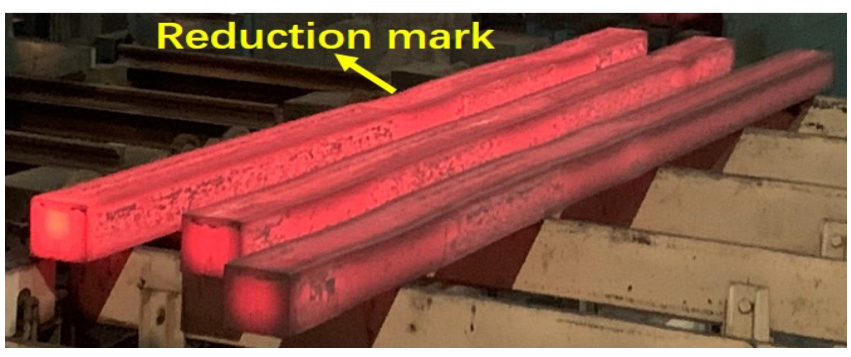

(a)

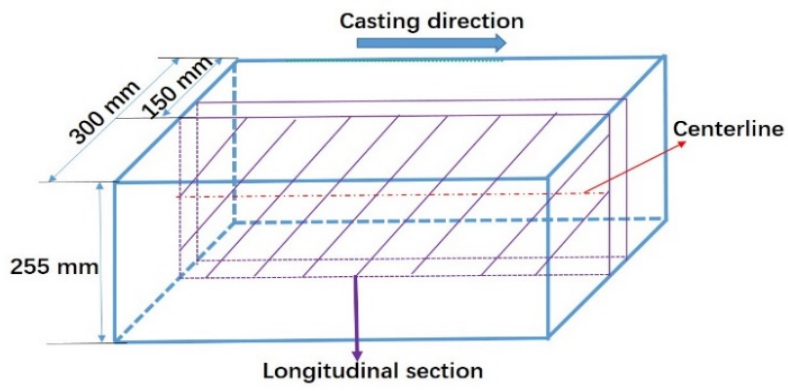

(b)

Figure 2. Reduction marks on bloom (a) and the bloom sampling plan for steel GCr15 (b).

Figure 3 shows the hot acid etching results of the longitudinal section corresponding to WS2 WS7 (the sample corresponding to WS1 was damaged). Obvious inner cracks and V-shaped segregation improvement could be observed at WS2 and WS3, which indicates that the fs at bloom center at the position of WS2 and WS3 would be in the range of 0.3 0.9. Slight V-shaped segregation improvement, but no inner cracks, at WS4 indicates where the solidification is close to the end. Neither inner cracks nor V-shaped segregation improvement was observed at WS5 WS7, which indicates that from the position of WS5 to WS7, the bloom was totally solidified where the fs at bloom center is equal to 1.0. The simulated bloom center solid fraction at WS1 WS7 is $0.39,0.60,0.82,0.98,1.00,1.00$, and 1.00 as shown in Figure 4, which agrees well with the "heavy reduction" experiment results when considering the inner crack occurrence position and the improvement extent of the V-shaped segregation improvement as the criterion of the bloom center solid fraction range. Thus, the solidification model also has a certain extent of accuracy when dealing with high-carbon steels. 

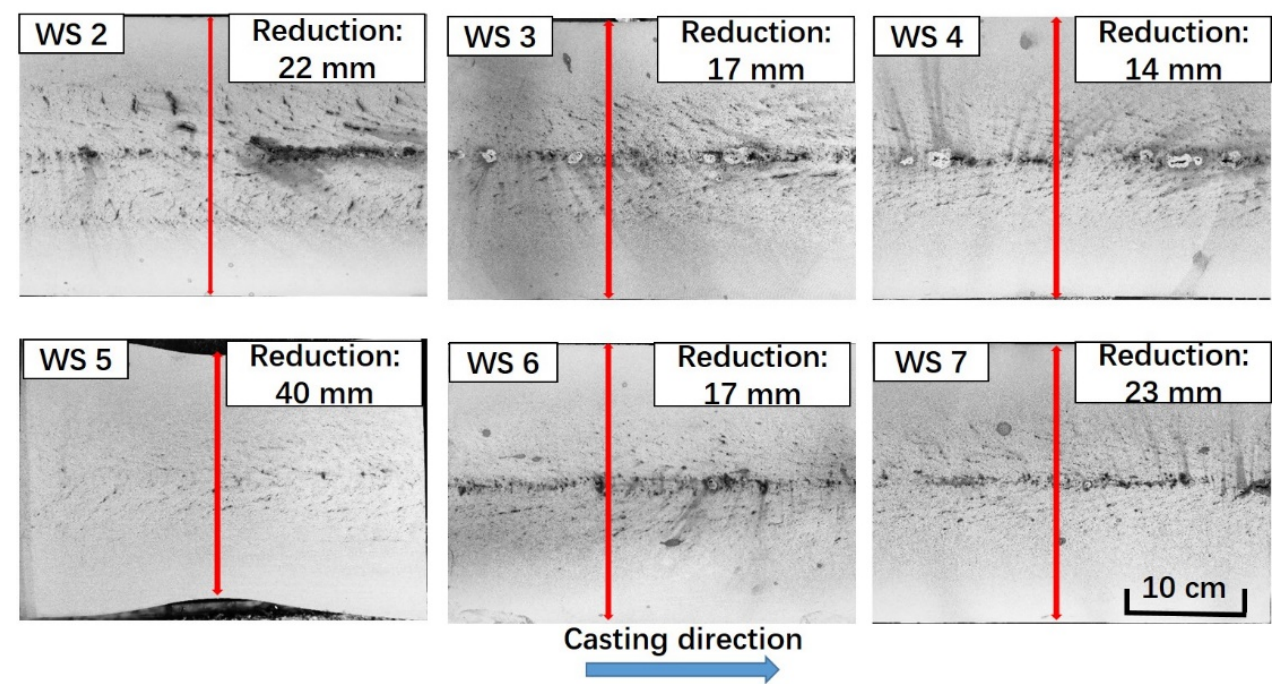

Figure 3. Hot etched photos of longitudinal section of the bearing steel blooms.

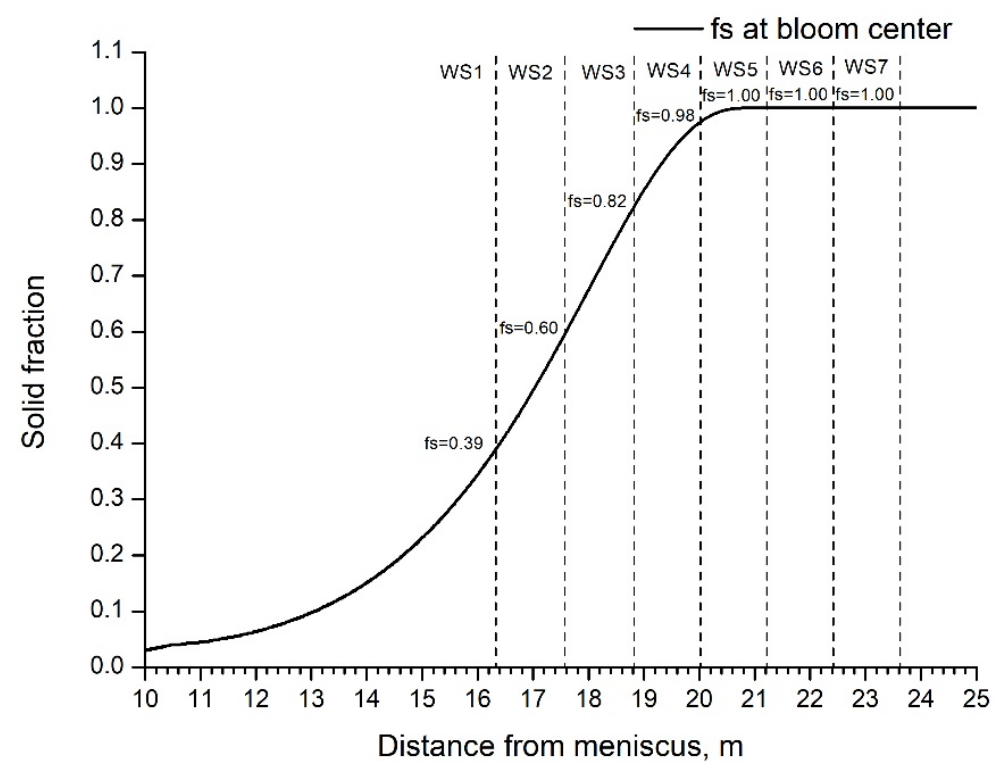

Figure 4. Simulated GCr15 bloom center solid fraction at different WS unit position.

\subsection{Model Application}

As mentioned earlier, the reasonable center solid fraction range along casting direction is $0.3 \sim 0.9$ for the MSR effect on segregation improvement of the internal quality of the bloom; thus, the determination of the position of the reduction zone and solidification crater by applying the simulation model is critical before industrial tests are conducted. The relationship between metallurgical length and casting speed, secondary cooling intensity (specific water volume, SWV), and casting superheat is mapped by playing with the model, as shown in Figure 5a. It can be seen that the metallurgical length would be prolonged from $21.40 \mathrm{~m}$ and $13.96 \mathrm{~m}$ to $22.71 \mathrm{~m}$ and $22.70 \mathrm{~m}$ when increasing casting superheat and speed from 10 to $50^{\circ} \mathrm{C}$ and 0.70 to $0.88 \mathrm{~m} / \mathrm{min}$, respectively. Meanwhile, when increasing the SWV from 0.10 to $0.37 \mathrm{l} / \mathrm{kg}$, the metallurgical length would be reduced from 22.24 to $21.64 \mathrm{~m}$. The simulation results indicate that, compared to secondary cooling intensity and casting superheat, casting speed has a much greater influence on the solidification crater.

Figure $5 \mathrm{~b}$ compares the solid fraction isolines, $\mathrm{fs}=0.0,0.3,0.9$, and 1.0 , in the bloom thickness direction at different casting speeds with the same secondary cooling intensity and superheat. When considering the center solid fraction range of $0.3 \sim 0.9$ as the effective MSR zone, it can be seen that the effective MSR zone is prolonged as casting speed increases. 
When casting speed is increased from 0.70 to $0.85 \mathrm{~m} / \mathrm{min}$, the effective MSR zone and metallurgical length is prolonged from 2.99 to $3.67 \mathrm{~m}$ and from 16.91 to $21.21 \mathrm{~m}$, respectively. From another perspective, when casting speed increases every $0.05 \mathrm{~m} / \mathrm{min}$, the effective MSR zone and metallurgical length is prolonged by about 0.23 and $1.43 \mathrm{~m}$, respectively. Moreover, unlike the slab casting, which usually has a dense roll pitch, the roll pitch of bloom caster is normally relatively large (more than $1 \mathrm{~m}$ ). If the number of reduction roller in the effective MSR zone is too small, the reduction of these WS units would be much larger than normal to provide enough reduction compensation to reduce segregation; however, this would increase the risk of inner crack occurrence. The reasonable solution should be adjusting casting parameters to have more WS units acting in the effective MSR zone, which could enable alternatives of different reduction distribution patterns for each MSR unit, as shown in Figure $5 \mathrm{~b}$ when the casting speed is $0.88 \mathrm{~m} / \mathrm{min}$.

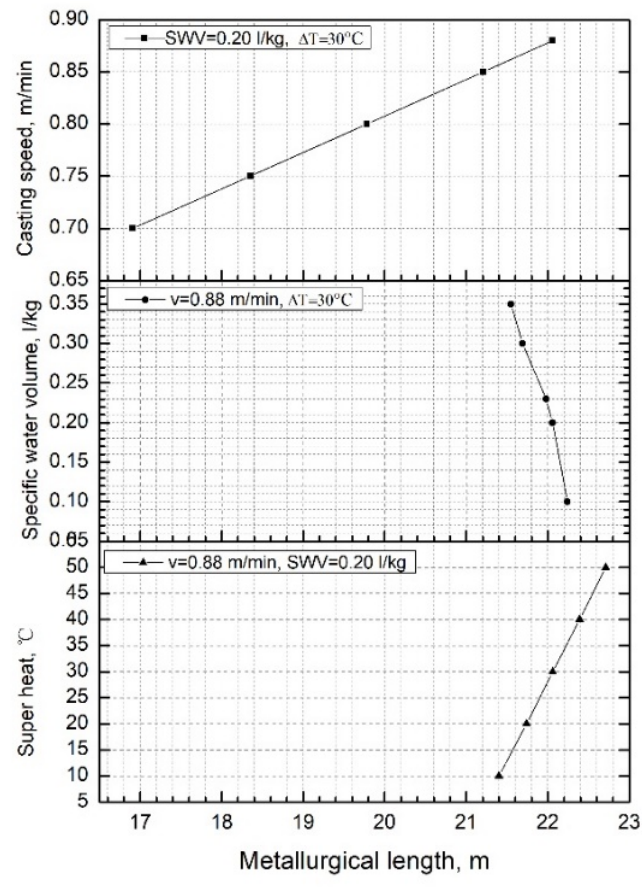

(a)

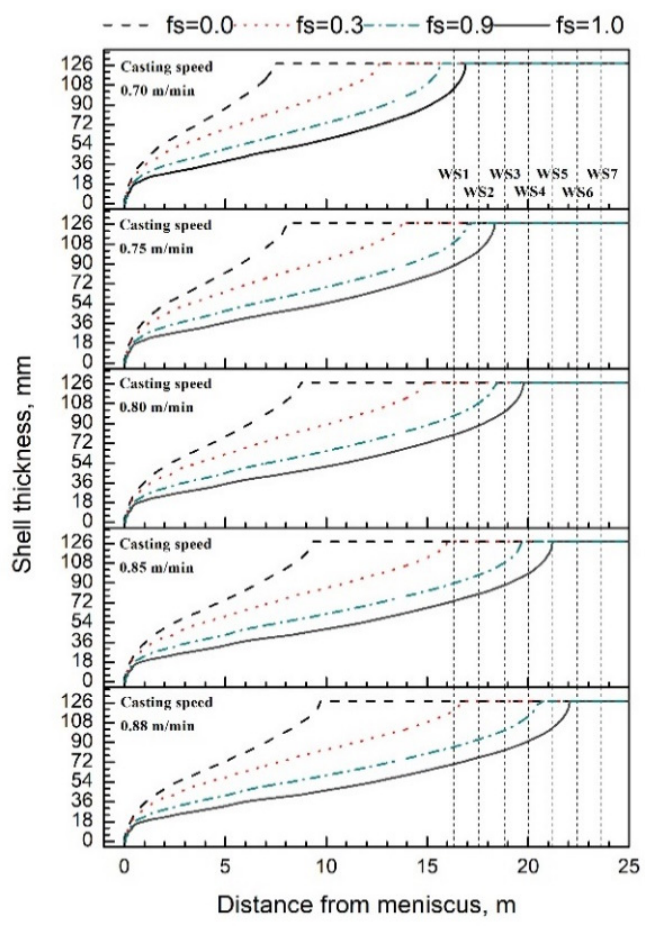

(b)

Figure 5. (a) Influence of casting condition on metallurgical length of steel GCr15; (b) solid fraction isolines at different casting speed of GCr15 steel.

\subsection{Caster Information and Experimental Scheme}

The 9\# bloom caster in NISCO was designed by SMS CONCAST and started up in March 2020. This four-strand, bow-type caster has a casting radius of $12 \mathrm{~m}$ and is equipped with an eddy-current mold lever monitoring system, mold electromagnetic stirring (MEMS) and F-MES, and mechanical sort and hard reduction (MSR and MHR). The bloom has a section of $255 \mathrm{~mm} \times 300 \mathrm{~mm}$, which is designed to match the subsequent medium-bar rolling mill. The main product of this caster is special steel, such as gear steel, construction machinery steel, nonquenched and tempered steel, spring steel, wire rod steel, bearing steel, etc. During the commissioning period, the bearing steel was taken as the critical steel grade to evaluate the performance of the caster. The detailed information of the 9\# caster and the chemical composition of the bearing steel is shown in Figure 6 and Table 1, respectively.

Based on the simulation results, several industrial trials were conducted regarding different casting speed (from 0.70 to $0.88 \mathrm{~m} / \mathrm{min}$ ) while the other casting conditions were the same. For each casting speed trial, several longitudinal samples were taken and cut out firstly to proceed with the hot deep acid etching test to reveal the V-shaped 
segregation, central porosity, and central segregation; the sampling plan is shown in Figure $2 \mathrm{~b}$. Thereafter, the samples are prepared for the segregation investigation by drilling method. The drilling point positions are shown in Figure 7. The drill has a diameter of $5 \mathrm{~mm}$, and the drill depth is around $8 \mathrm{~mm}$. The drilling cuttings are analyzed by the carbon-sulfur analyzer. As shown in Figure 7, the drilling points of 1 10 are randomly chosen and the average carbon content of these 10 points is taken as the average carbon content, $\mathbf{c}_{0}$, of this sample. The rest of the drilling points, which have an interval of $20 \mathrm{~mm}$, are the central points of the bloom. The carbon segregation index is defined by taking the carbon content, $\mathbf{c}$, at each drilling point of the bloom centerline divided by the average carbon content of the 10 random chosen drilling points, i.e., carbon segregation index equals $\mathbf{c} / \mathbf{c}_{\mathbf{0}}$.

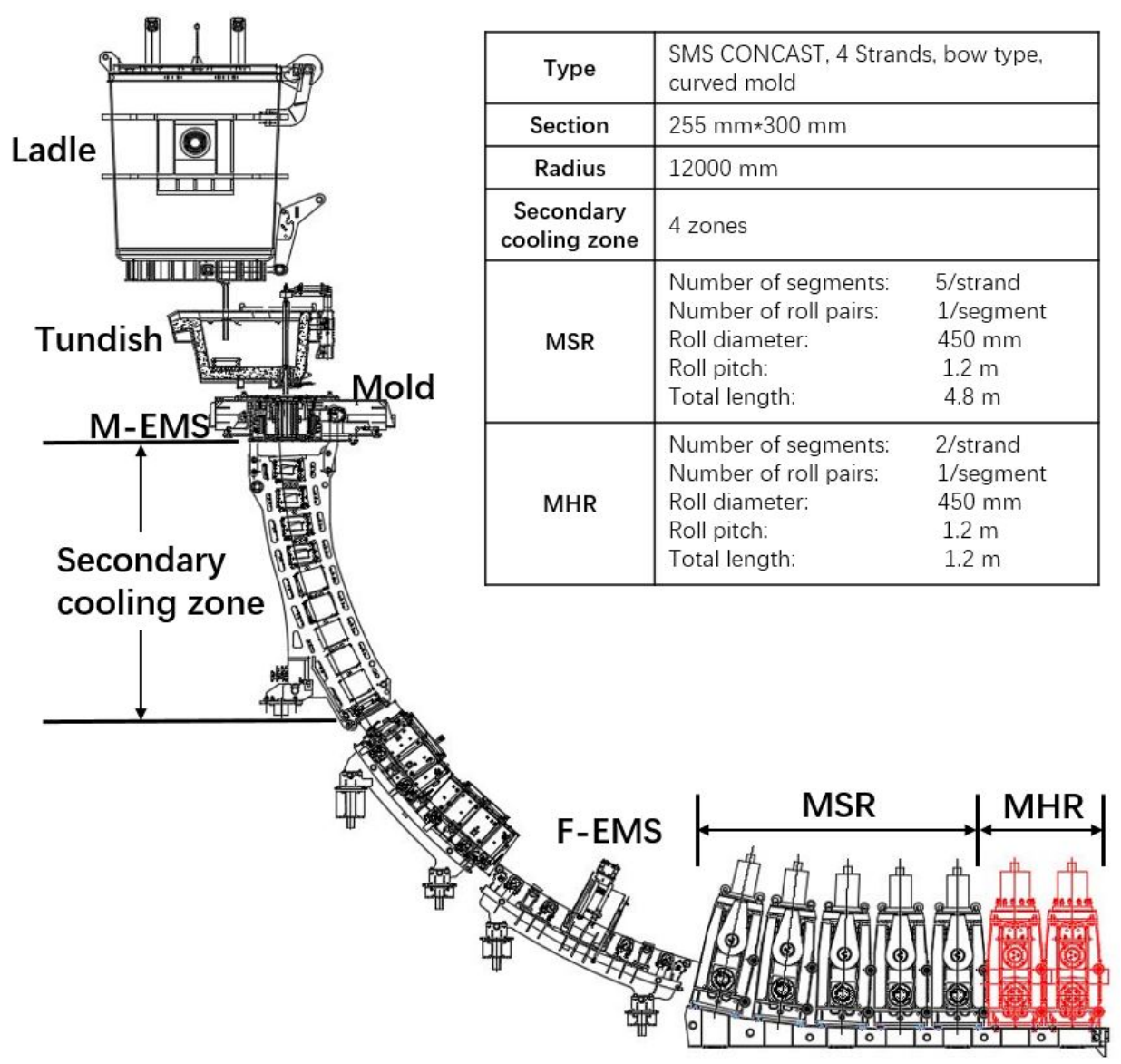

Figure 6. Layout of the 9\# bloom caster and its technical parameters.

Table 1. Chemical composition ${ }^{1}$ of the tested bearing steel, wt. $\%$.

\begin{tabular}{cccccccc}
\hline Steel Grade & C & Si & Mn & P & S & Cr & Al \\
\hline GCr15 & $0.95 \sim 1.00$ & $0.20 \sim 0.30$ & $0.30 \sim 0.40$ & $\leq 0.020$ & $\leq 0.015$ & $1.40 \sim 1.50$ & $0.010 \sim 0.030$ \\
\hline
\end{tabular}

${ }^{1}$ Measured from tundish. 


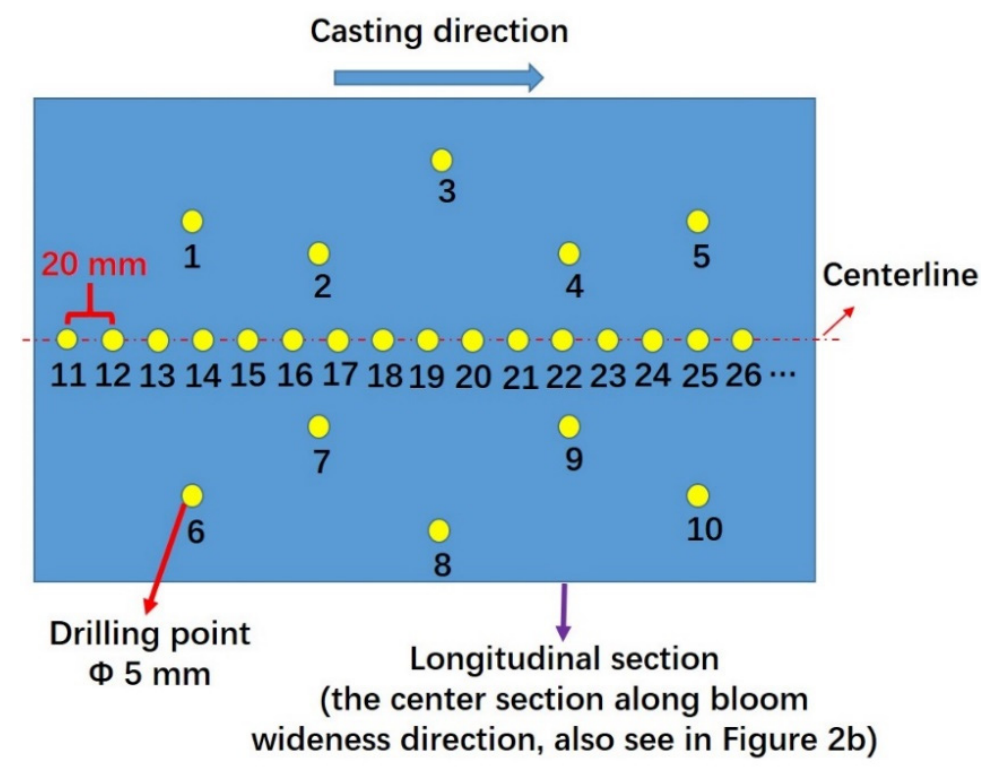

Figure 7. Drilling point positions of longitudinal section for segregation evaluation.

\section{Results and Discussions}

Industrial trials with different casting speed were conducted in NISCO to optimize the MSR parameters regarding the improvement of internal quality of the bearing steel blooms. The trial parameters are listed in Table 2 . The tested casting speed ranged from 0.70 to $0.88 \mathrm{~m} / \mathrm{min}$, with the effective reduction amount from 0 to $8.5 \mathrm{~mm}$. Based on the simulation results and the MSR roll pitch of $1.2 \mathrm{~m}$, the calculated reduction rate of the trials ranged from $0 \sim 4.6 \mathrm{~mm} / \mathrm{m}$ and $0 \sim 3.7 \mathrm{~mm} / \mathrm{min}$, respectively.

Table 2. Trial casting parameters of steel GCr15.

\begin{tabular}{cccccc}
\hline Trial No. & $\mathbf{a}$ & $\mathbf{b}$ & $\mathbf{c}$ & $\mathbf{d}$ & $\mathbf{e}$ \\
\hline Casting speed, $\mathrm{m} / \mathrm{min}$ & 0.70 & 0.75 & 0.80 & 0.85 & 0.88 \\
MSR amount, mm, fs $=0.3 \sim 0.9$ & 0 & 2 & 5.5 & 8 & 8.5 \\
MHR amount, $\mathrm{mm}, \mathrm{fs}=1.0$ & 0 & 20 & 11 & 15 & 19 \\
MSR reduction rate, $\mathrm{mm} / \mathrm{m}$ & 0 & 1.7 & 4.6 & 3.3 & 2.4 \\
MSR reduction rate, $\mathrm{mm} / \mathrm{min}$ & 0 & 1.3 & 3.7 & 2.8 & 2.1 \\
Total reduction amount, $\mathrm{mm}$ & 0 & 28 & 27 & 33 & 29.5 \\
\hline
\end{tabular}

Figure 8 shows the hot etched photos of the longitudinal section of the trials. Due to the effective MSR zone of trial a being ahead of the WS 1 when the casting speed is $0.70 \mathrm{~m} / \mathrm{min}$, the MSR will not affect the compensation of the solidification shrinkage; thus, no MSR was applied for trial a. Obvious V-shaped segregation lines, center porosities, continuous center segregation, and mini-ingot are clearly observed at casting speed of $0.70 \mathrm{~m} / \mathrm{min}$, which is similar to the conventional ingot solidification. As casting speed increases to $0.75,0.80$, and $0.85 \mathrm{~m} / \mathrm{min}$, in trial $\mathbf{b}, \mathbf{c}$, and $\mathbf{d}$, the number of WS units located in the effective MSR zone also increases from 1 to 3 . This gives the reasonable process window for MSR to function effectively. The higher the casting speed, the better the internal quality of the bloom. However, the solidification defects are still severe, which reveals that the compensation of the solidification shrinkage by MSR is still not enough. In the case of casting speed of $0.85 \mathrm{~m} / \mathrm{min}$, in trial $\mathbf{d}$, V-shaped segregation lines could be barely observed at the center region of the bloom; however, due to the relatively large reduction rate in the straightening zone (from WS1 to WS4), reduction cracks occurred. When further increasing the casting speed to $0.88 \mathrm{~m} / \mathrm{min}$, in trial $\mathbf{e}$, better internal quality without reduction cracks was obtained. Compared with trial $\mathbf{d}$, due to the prolonged effective MSR zone, although the MSR amount was increased, the reduction rate was reduced, and this could be the reason for the diminishing occurrence of reduction cracks. It should be mentioned that 
trials $\mathbf{b}-\mathbf{e}$ were all applied with MHR. The optimization of the MHR was not discussed here, as the heavy reduction was conducted only by WS7 where the solidification was already finished and could not affect the compensation during solidification. The applied MHR values were provided only for reference.

It can be seen that the internal quality of the bloom center region of trial $\mathbf{e}$ is the best among all the conducted trials. The results reveal that from the aspect of solidification macrostructure, favorable internal quality of the bearing steel can be obtained by reasonable MSR reduction rate.

The comparative evaluation of the influence of the soft reduction process at different casting conditions on the center segregation level along casting direction is given in Table 3 and Figure 9. It can be seen that increasing the effective MSR zone length and the MSR reduction amount leads to a lower and smaller deviation of the center segregation index, indicating a more homogeneous segregation level of the bloom. In trial e, casting speed is $0.88 \mathrm{~m} / \mathrm{min}$, which shows the best segregation degree for which the mean center carbon segregation index and ratio of segregation index between $0.92 \sim 1.08$ is 1.00 and $97 \%$, respectively.

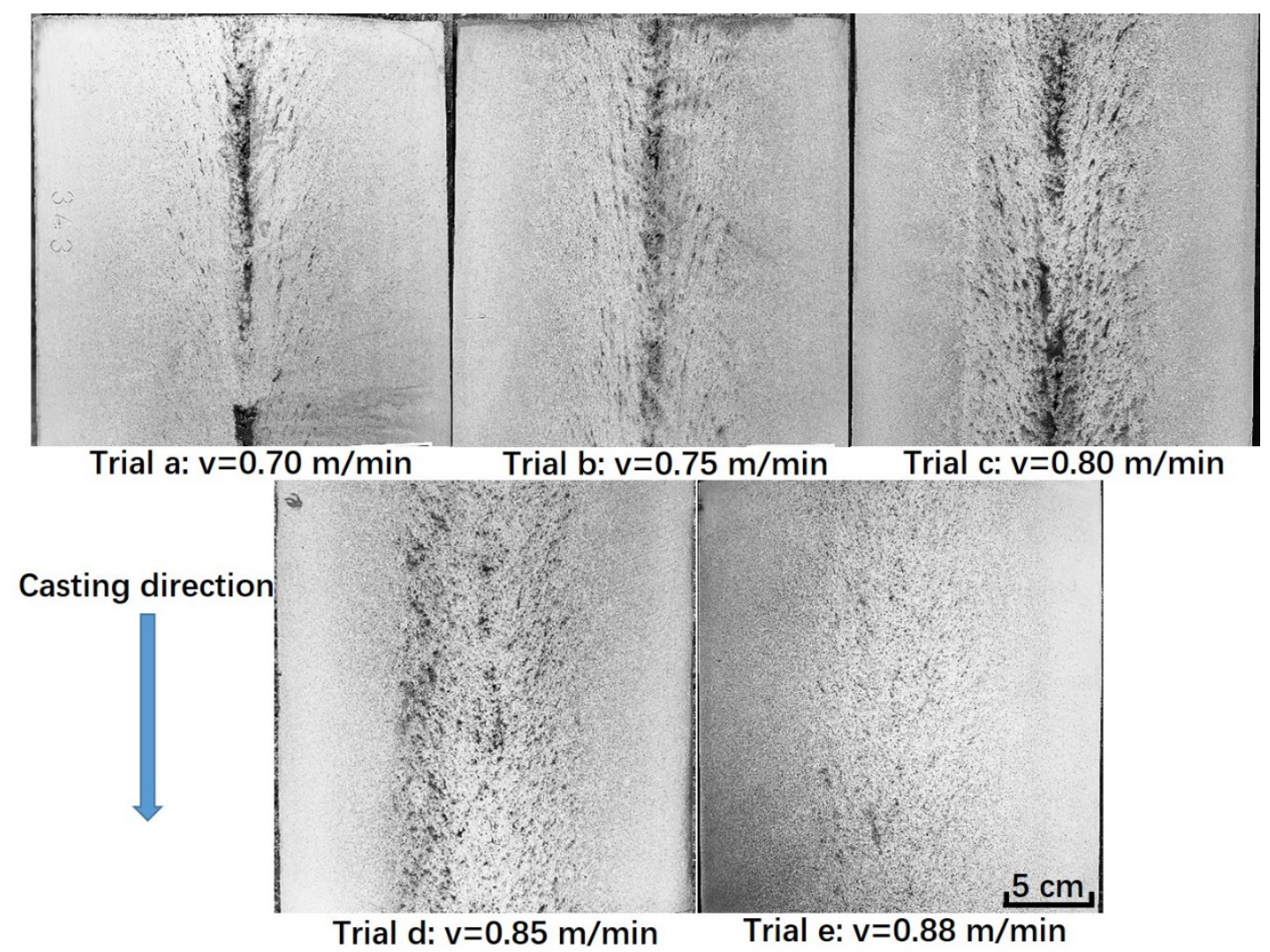

Figure 8. Hot etched bloom longitudinal sections of different casting speed trials.

Table 3. Bloom center carbon segregation level at different casting conditions.

\begin{tabular}{|c|c|c|c|c|c|}
\hline $\begin{array}{l}\text { Casting Speed, } \\
\mathrm{m} / \mathrm{min}\end{array}$ & $\begin{array}{c}\text { Center Seg. }{ }^{2} \text { Data } \\
\text { Quantity }\end{array}$ & Max. Seg. ${ }^{2}$ Index & Min. Seg. ${ }^{2}$ Index & Mean Seg. ${ }^{2}$ Index & $\begin{array}{c}\text { Ratio of Seg. }{ }^{2} \\
\text { Index between } \\
0.92 \sim 1.08\end{array}$ \\
\hline 0.70 & 39 & 1.23 & 0.90 & 1.05 & $67 \%$ \\
\hline 0.75 & 36 & 1.29 & 0.91 & 1.12 & $31 \%$ \\
\hline 0.80 & 111 & 1.30 & 0.93 & 1.06 & $63 \%$ \\
\hline 0.85 & 132 & 1.15 & 0.93 & 1.02 & $89 \%$ \\
\hline 0.88 & 163 & 1.11 & 0.91 & 1.00 & $97 \%$ \\
\hline
\end{tabular}

${ }^{2}$ Seg. is "Segregation" for short. 


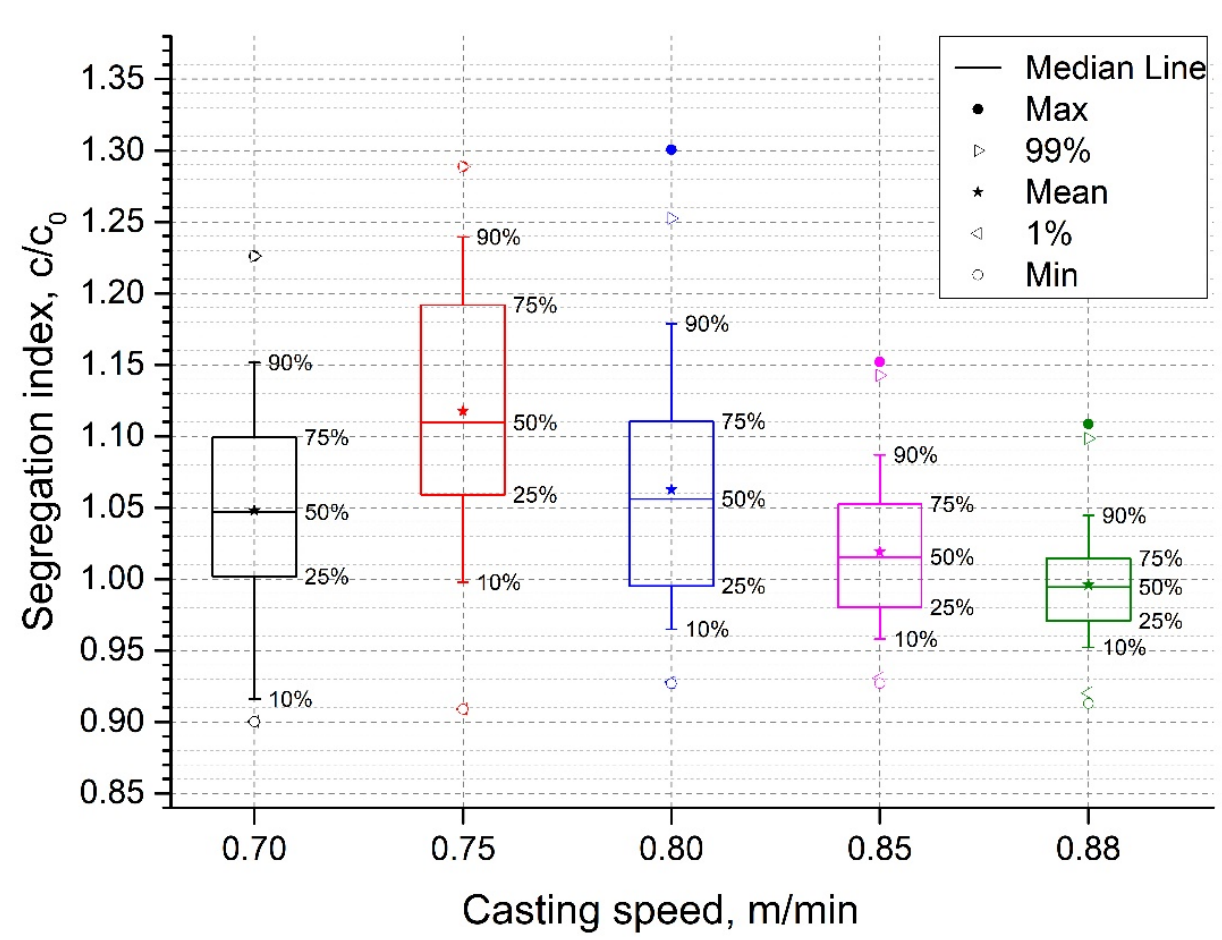

Figure 9. Statistical distribution of the bloom center carbon segregation level at different casting conditions for steel GCr15.

\section{Conclusions}

Extensive industrial MSR trials with different reduction rates were successfully carried out and discussed regarding the improvement of the internal quality of the bearing steel bloom. The simulation model provided by SMS CONCAST has a certain accuracy for both medium (45\#)- and high-carbon (GCr15) steels, which was verified by the developed "white band" and "heavy reduction" methods. The simulation results indicate that, compared to secondary cooling intensity and casting superheat, casting speed has a much greater influence on the solidification crater. It is evident that the MSR reduction rate and effective MSR zone length have great influence on the internal quality of the bearing steel bloom. From the aspects of solidification macrostructure and center carbon segregation homogeneity, the suitable casting condition for bearing steel (GCr15) should have a casting speed of $0.88 \mathrm{~m} / \mathrm{min}$ and MSR rate of $2.4 \mathrm{~mm} / \mathrm{m}$ or $2.1 \mathrm{~mm} / \mathrm{min}$ for the $9 \#$ caster in NISCO. Due to the limitation of the BOF-CCmatching and production organization in NISCO, trials with higher casting speed were not conducted. However, it could be deduced that further increasing the casting speed would bring a more reasonable reduction distribution pattern for the MSR application during the continuous casting of bearing steel blooms.

Author Contributions: Conceptualization, X.C. and S.N.; methodology, W.D.; software, S.N.; validation, X.C., W.D., and S.N.; formal analysis, S.N.; investigation, W.D.; resources, X.C.; data curation, W.D.; writing-original draft preparation, X.C.; writing—review and editing, S.N.; visualization, W.D.; supervision, X.C.; project administration, S.N.; funding acquisition, X.C. All authors have read and agreed to the published version of the manuscript.

Funding: This research received no external funding.

Institutional Review Board Statement: Not applicable.

Informed Consent Statement: Not applicable.

Conflicts of Interest: The authors declare no conflict of interest. 


\section{References}

1. Krauss, G. Solidification, segregation, and banding in carbon and alloy steels. Metall. Mater. Trans. B 2003, 34, 781-792. [CrossRef]

2. Bode, O.; Schwerdtfeger, K.; Geck, H.G.; Höfer, F. Influence of casting parameters on void volume and centre segregation in continuously cast 100Cr6 blooms. Ironmak. Steelmak. 2008, 35, 137-145. [CrossRef]

3. Sun, J.; Sheng, C.; Wang, D.P.; Zhang, Y.H.; Zhong, H.G.; Xu, Z.S.; Li, L.J.; Zhai, Q.J. Influence of pulsed magneto-oscillation on microstructure and mechanical property of rectangular 65Mn steel ingot. J. Iron Steel Res. Int. 2018, 25, 862-866. [CrossRef]

4. Cui, L.X.; Lei, X.H.; Zhang, L.F.; Zhang, Y.X.; Yang, W.; Gao, Y.; Liu, Y.B.; Liu, N. Three-Dimensional Characterization of Defects in Continuous Casting Blooms of Heavy Rail Steel Using X-ray Computed Tomography. Metall. Mater. Trans. B 2021, 52, 2327-2340. [CrossRef]

5. El-Bealy, M.O. Macrosegregation quality criteria and mechanical soft reduction for central quality problems in continuous casting of steel. Mater. Sci. Appl. 2014, 5, 724-744. [CrossRef]

6. Wu, M.H.; Kharicha, A.; Ludwig, A. Discussion on modeling capability for macrosegregation. High Temp. Mater. Proc. 2017, 36, 531-539. [CrossRef]

7. Bleck, W.; Wang, W.; Rülte, R. Influence of soft reduction on internal quality of high carbon steel billets. Steel Res. Int. 2006, 77, 485-491. [CrossRef]

8. Li, B.; Zhang, Z.H.; Liu, H.S.; Luo, M.; Lan, P.; Tang, H.Y.; Zhang, J.Q. Characteristics and evolution of the spot segregations and banded defects in high strength corrosion resistant tube steel. Acta Metall. Sin. 2019, 55, 762-772.

9. Zhang, Z.; LI, H.Y.; Zhou, L.; Liu, H.S.; Tang, H.Y.; Zhang, J.Q. As-Cast Spot Segregation of gear steel and Its evolution in the rolled products. Acta Metall. Sin. 2021, 57, 1281-1290.

10. Tomono, H.; Hitomi, Y.; Ura, S.; Teraguchi, A.; Iwata, K.; Yasumoto, K. Mechanism of formation of the v-shaped segregation in the large section continuous cast bloom. Trans. ISIJ 1984, 24, 917-922. [CrossRef]

11. Thome, R.; Harste, K. Principles of billet soft-reduction and consequences for continuous casting. ISIJ Int. 2006, 46, 1839-1844. [CrossRef]

12. Suzuki, M.; Kimura, K.; Kawami, A.; Mizutani, M. Improvement in center segregation of high carbon steel continuous casting blooms. Rev. Met. Paris 1992, 89, 83-92. [CrossRef]

13. Thome, R.; Harste, K. Study on the design of the soft reduction unit of high-speed billet casters. Steel Res. Int. 2004, 75, 693-700. [CrossRef]

14. Thome, R.; Ostheimer, V.; Ney, G. Soft reduction in the continuous casting of billets. Millennium Steel 2007, 112-118.

15. Ji, C.; Luo, S.; Zhu, M. Analysis and application of soft reduction amount for bloom continuous casting process. ISIJ Int. 2014, 54, 504-510. [CrossRef]

16. Luo, S.; Zhu, M.; Ji, C. Theoretical model for determining optimum soft reduction zone of continuous casting steel. Ironmak. Steelmak. 2014, 41, 233-240. [CrossRef]

17. Li, L.; Zhang, Z.H.; Luo, M.; Li, B.; Lan, P.; Zhang, J.Q. Control of shrinkage porosity and spot segregation in $\varnothing 195 \mathrm{~mm}$ continuously cast round bloom of oil pipe steel by soft reduction. Metals 2021, 11, 9. [CrossRef]

18. Bridge, M.R.; Rogers, G.D. Structural effects and band segregate formation during the electromagnetic stirring of strand-cast steel. Metall. Mater. Trans. B 1984, 15, 581-589. [CrossRef]

19. Kojima, S.; Mizota, H. Quantitative analysis for center segregation of continuously cast slabs. Tetsu-to-Hagane 1983, 69, 263.

20. Fujii, H.; Ohashi, T.; Hiromoto, T. On the formation of internal cracks in continuously cast slabs. Trans. ISIJ 1978, 18, 510-518. [CrossRef]

21. Wolf, M. Strand cast halfway cracks-How to read a suphur print. In Proceedings of the 82nd Steelmaking Conference, Chicago, IL, USA, 21-24 March 1999; pp. 3-10.

22. Takahashi, T.; Kudoh, M.; Ichikawa, K. Fluidity of the liquid in the solid-liquid coexisting zone. Trans. Jpn. Inst. Met. 1980, 21, 531-538. [CrossRef] 